Letter to the Editor

\title{
Novel use of adjunctive hyperbaric therapy for fungal scleritis
}

\author{
Harry Yip MBBS ${ }^{1,2}$ and Elsie Chan FRANZCO ${ }^{1,3,4}$
}

1. Royal Victorian Eye and Ear Hospital, Melbourne, Australia

2. Alfred Hospital, Melbourne, Australia

3. Ophthalmology, Department of Surgery, University of Melbourne, Melbourne, Australia

4. Centre for Eye Research Australia, Melbourne, Australia

Correspondence: Dr Harry Yip, Royal Victorian Eye and Ear Hospital, 32 Gisborne St East Melbourne, VIC 3002, Australia

Email: harry.yip.au@gmail.com

Received 9 September 2019; accepted 31 November 2019

Funding sources / Financial disclosure: None

Conflict of interest: None

This is the author manuscript accepted for publication and has undergone full peer review but has not been through the copyediting, typesetting, pagination and proofreading process, which may lead to differences between this version and the Version of Record. Please cite this article as doi: $10.1111 /$ ceo.13689

This article is protected by copyright. All rights reserved. 
Fungal scleritis is a rare condition that is difficult to treat due to delays in diagnosis, the aggressive nature of causative organisms and associated scleral ischaemia or necrosis. ${ }^{1}$ Conventional management of fungal scleritis includes prompt surgical debridement of the affected area in addition to empirical anti-microbials until targeted, culture-based therapy can be started. ${ }^{1}$

Hyperbaric oxygen therapy (HBOT) is a treatment that aims to improve wound healing through hyper-oxygenation. ${ }^{2}$ In ophthalmology, HBOT is used for infections not responding to conventional management and ocular conditions involving

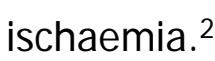

We report a case of the novel use of HBOT as an adjunct to treat Cladosporium fungal scleritis secondary to pterygium removal with topical mitomycin $\mathrm{C}$. This is only the second reported case of HBOT use in fungal scleritis and the first case where it was used with resolution of the infection. ${ }^{3}$

A 45-year old female presented 1 month following excision of a right nasal pterygium with mitomycin C. Visual acuity was $6 / 15$ in the right eye. On slit-lamp examination, the sclera was avascular with an overlying conjunctival defect and corneal thinning around the nasal limbus. The patient had no significant past ocular or medical history, recent overseas travel or contact with a rural environment, and serology for autoimmune markers was negative. After a few months of topical lubricants, the conjunctival defect healed. 
A year later, the patient returned with a recurrent and persistent conjunctival defect which was managed with a conjunctival autograft. At this time, there was no recent use of topical steroid treatment. Following surgery, the patient was started on topical Prednefrin $\circledast$ Forte (prednisolone acetate $1 \%$ + phenylephrine hydrochloride $0.12 \%$, Allergan, NSW). The autograft retracted and a scleral plaque developed. The plaque was surgically removed, and a second conjunctival autograft was placed on the area of resection. Histology of the plaque showed dystrophic calcification."

Seven weeks following the plaque removal, the patient represented with pain. The sclera appeared thickened and the overlying conjunctiva was inflamed, but there was no overlying epithelial defect. The anterior chamber had $1+$ cells and the posterior segment examination was unremarkable. The patient had been on regular topical steroid drops over the preceding 7 months, since surgery for the conjunctival autograft. A diagnosis of non-infectious anterior scleritis was made and the patient commenced oral prednisolone 50mg daily.

Three days later, the patient returned with worsening pain and a visual acuity of 'hand movements'. A nasal corneal infiltrate was noted. The diagnosis was modified to infectious sclerokeratitis. A corneal scrape and repeated scleral biopsies were performed, which were negative on microbiology. The patient subsequently had a corneal perforation which was managed with cyanoacrylate glue (Histoacryl ${ }^{\circledR}$, B. Braun Australia Pty Ltd), and the necrotic sclera was surgically debrided. The patient was started on cefazolin as prophylaxis against bacterial infection. Due to the suspicion of a fungal cause, a single dose of intrastromal and subconjunctival voriconazole $(50 \mu \mathrm{g} / \mathrm{mL}$ ) were administered, and topical $1 \%$ voriconazole every hour, topical $5 \%$ natamycin, oral voriconazole $300 \mathrm{mg}$ and terbanafine $250 \mathrm{mg}$ twice daily were commenced. 
Two weeks after the diagnosis of infectious sclerokeratitis, the fungal polymerase chain reaction (PCR) from the scleral biopsies detected Cladosporium sp.. The patient's antimicrobial regime was changed to oral posaconazole 300mg daily in addition to topical voriconazole and topical $0.15 \%$ amphotericin $B$ hourly with prophylactic topical ofloxacin $0.3 \%$. All other antimicrobials were ceased. As the patient had received a fortnight of medical treatment with minimal improvement, the decision to utilise adjunctive HBOT was made, which was commenced the next day. A HBOT schedule of 1-hourly sessions 5 times a week, totalling 40 sessions over 2 months was organised.

Figure 1: Right eye prior to HBOT. There was a large area of avascular, thin scleral defect associated with an adjacent corneal infiltration.

In total, the patient received 3 months of treatment with amphotericin B eye drops, 3 months of prophylactic ofloxacin eye drops, 6 months of oral posaconazole, 11 months of voriconazole eye drops and 40 sessions of HBOT. On review 3 months after cessation of all anti-fungal treatment, there was resolution of the corneal infiltrate, and re-epithelialization and revascularization over the sclera (Figure 2). The glue covering the corneal perforation was removed 15 months after it was applied. Eleven months after ceasing anti-fungal treatment there has been no recurrence of infection. The visual acuity was $6 / 60$ in the presence of a nuclear cataract.

Figure 2: Right eye post-HBOT (with cryanoacrylate glue in situ).

HBOT use in ophthalmology has been driven by biochemical and microbiological principles, with some supportive evidence from case series and studies. ${ }^{2}$ The only previously published case of HBOT use in infectious scleritis was a Rhizopus fungal 
scleritis in which conventional anti-fungal therapy and adjunctive HBOT were unsuccessful in treating the infection, resulting in enucleation. ${ }^{3}$

Use of HBOT in infective ocular conditions such as keratitis can be considered when primary therapy fails. Chong et al. reported the use of adjunctive HBOT in a patient with Pseudomonas keratitis which was not responsive to topical, oral and intravenous antibiotics. ${ }^{4}$ HBOT was used due to its known efficacy in the treatment of Pseudomonas malignant otitis externa and the success in using HBOT to treat Pseudomonas infections in vitro and in vivo. ${ }^{4}$ Following the use of HBOT, the patients visual acuity improved from $6 / 12$ to $6 / 6$ with mild corneal thinning and scarring. ${ }^{4}$

The use of HBOT has also been reported in ocular conditions involving ischaemia; including retinal artery and vein occlusions, anterior segment ischaemia and scleral melt. ${ }^{2}$ In scleral ischaemia, HBOT is proposed to stimulate scleral angiogenesis and fibroblast proliferation. ${ }^{5}$ Bayer et al. demonstrated HBOT use in a necrotic sclera secondary to mitomycin- $\mathrm{C}$ treatment. ${ }^{5}$ After failing conventional management, HBOT use resulted in revascularisation and re-epithelialisation of the sclera. ${ }^{5}$ Similarly, our case suggests that HBOT may be beneficial in such cases, with our patient's avascular scleral defect improving following treatment.

HBOT can cause changes in refraction and induce nuclear cataract through oxidative stress on lens proteins. ${ }^{2}$ The refractive change is commonly a myopic shift during treatment which may resolve when treatment is ceased. ${ }^{2}$ Clinicians should be aware of these side effects when monitoring visual acuity in HBOT patients.

This is the first reported case of HBOT used successfully as an adjunctive treatment following failure of conventional treatment in Cladosporium scleritis. Extended antifungal treatment and early surgical intervention are still the gold standard of 
treatment for fungal scleritis. However, our report suggests that HBOT may have some utility as an adjunctive treatment of infective conditions of the eye. 


\section{REFERENCES}

1. Ramenaden ER, Raiji VR. Clinical characteristics and visual outcomes in infectious scleritis: a review. Clin Ophthalmol. 2013;7:2113-22.

2. Oguz H, Sobaci G. The use of hyperbaric oxygen therapy in ophthalmology. Surv Ophthalmol. 2008;53(2):112-20.

3. Locher DH, Adesina A, Wolf TC, Imes CB, Chodosh J. Postoperative Rhizopus scleritis in a diabetic man. J Cataract Refract Surg. 1998;24(4):562-5.

4. Chong R, Ayer CJ, Francis IC, Coroneo MT, Wolfers DL. Adjunctive hyperbaric oxygen in pseudomonas keratitis. Br J Ophthalmol. 2007;91(4):560-1.

5. Bayer A, Mutlu FM, Sobaci G. Hyperbaric oxygen therapy for mitomycin Cinduced scleral necrosis. Ophthalmic Surg Lasers. 2002;33(1):58-61. 


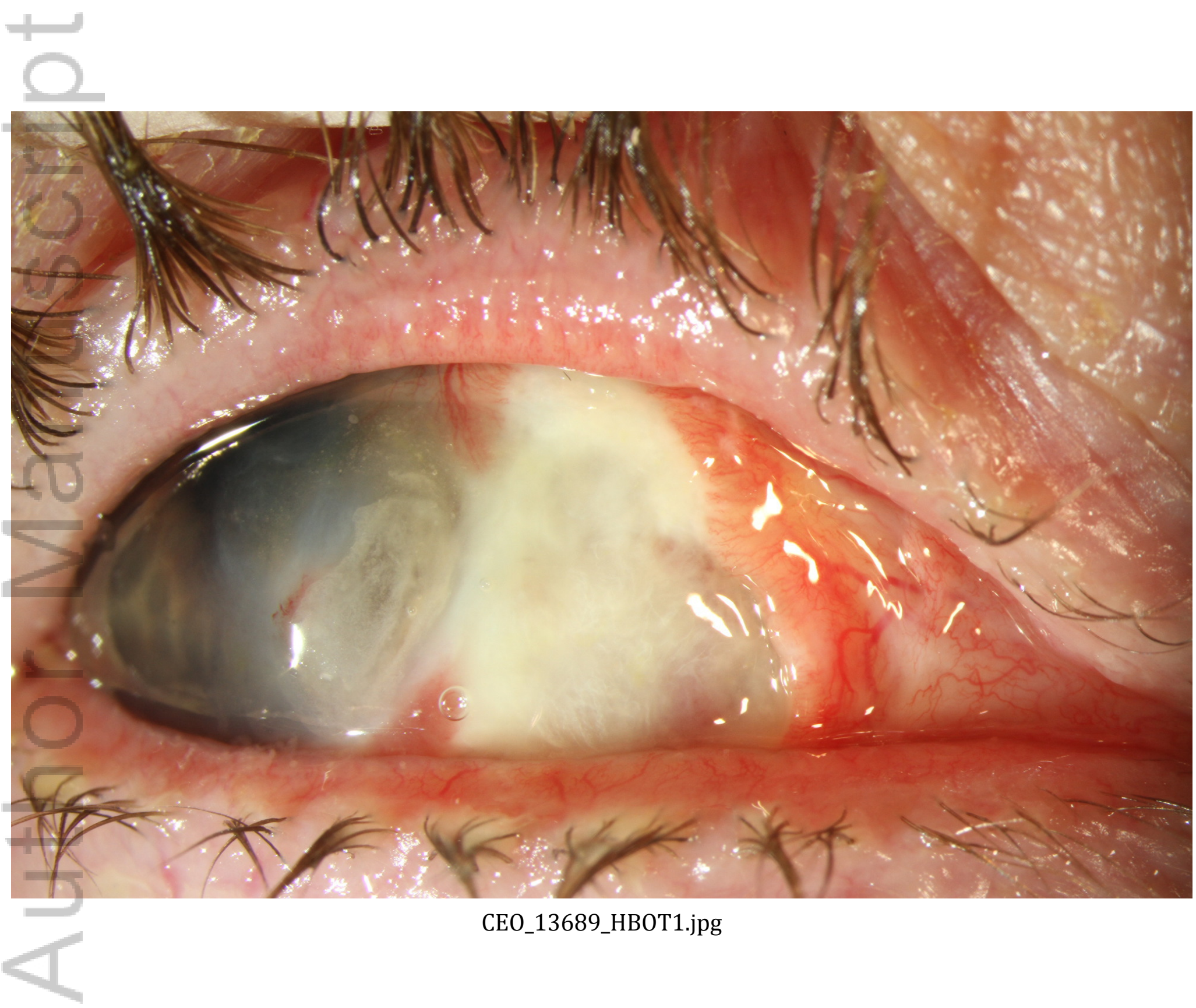

This article is protected by copyright. All rights reserved. 


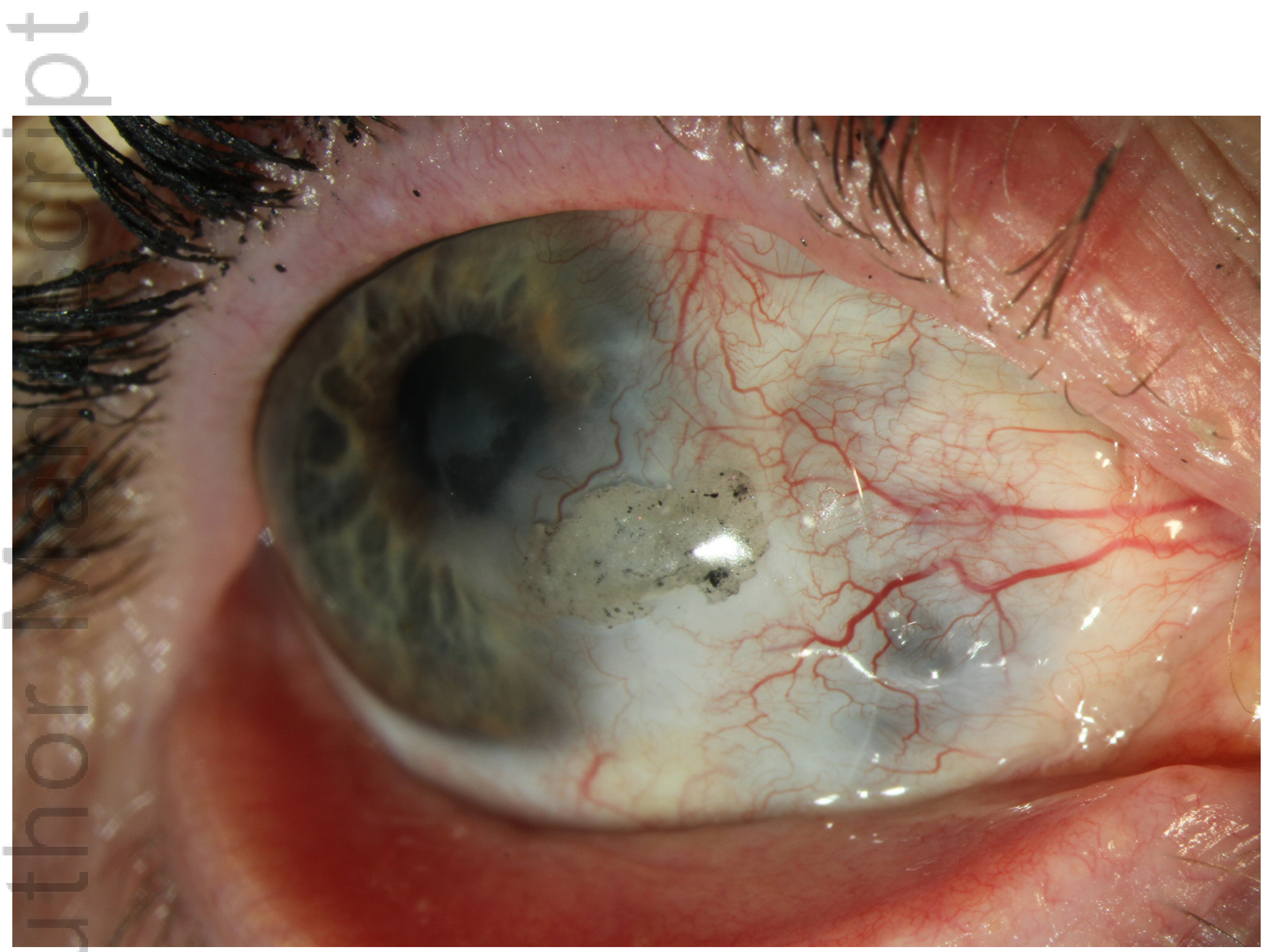

CEO_13689_HBOT2.jpg

This article is protected by copyright. All rights reserved. 


\section{University Library}

\section{- M M I N E R VA A gateway to Melbourne's research publications}

Minerva Access is the Institutional Repository of The University of Melbourne

Author/s:

Yip, H;Chan, E

Title:

Novel use of adjunctive hyperbaric therapy for fungal scleritis

Date:

2019-12-06

Citation:

Yip, H. \& Chan, E. (2019). Novel use of adjunctive hyperbaric therapy for fungal scleritis. CLINICAL AND EXPERIMENTAL OPHTHALMOLOGY, 48 (3), pp.404-406. https:// doi.org/10.1111/ceo.13689.

Persistent Link:

http://hdl.handle.net/11343/286717 